\title{
Relationship between a single bout of exhaustive exercise and endoplasmic reticulum stress in skeletal muscle
}

\author{
M. Xiang ${ }^{1}$, Y. Wen ${ }^{2}$, H. $\mathrm{Ai}^{2}$ and L. S. Harbige ${ }^{1}$ \\ ${ }^{1}$ Centre for Biosciences Research, School of Science, University of Greenwich, Kent ME4 4TB, UK and ${ }^{2}$ Division of \\ Nutrition and Biochemistry, Institute of Sports Medicine, The Third Hospital, Peking University, Beijing, P.R. China
}

The endoplasmic reticulum (ER) is arranged in a dynamic tubular network involved in metabolic processes, such as gluconeogenesis and lipid synthesis. Initial protein maturation steps that take place at the ER are crucial for the proper folding of proteins that are synthesized in the secretory pathway, which amount to approximately $30 \%$ of the total proteome in most eukaryotic cells. The protein-folding machinery in the ER is particularly challenged in specialized secretory cells owing to their high demand for protein synthesis, which constitutes a constant source of stress ${ }^{(1)}$. ER responds to the accumulation of unfolded proteins in its lumen (ER stress) by activating intracellular signal transduction pathways-cumulatively called the unfolded protein response (UPR) ${ }^{(2)}$. Novel, unaccustomed exercise has been shown to result in temporary, repairable skeletal muscle damage. After exhaustive endurance exercise, muscle damage can be produced by metabolic disturbances associated with ischaemia. Extensive disruption of muscle fibres also occurs after relatively short term eccentric exercise where high mechanical forces are generated. Rodent models of exercise can serve as useful models to study the exercise-dependent regulation of signal transduction pathways, gene expression and protein levels in the muscle ${ }^{(3)}$. The present study investigated the effect of a single bout of exhaustive exercise on ER stress biomarkers at gene and protein levels in quadriceps from a single bout of exhaustive swimming in C57/BL6 male mice. It was found that the levels of glucose-regulated protein 78 (GRP78), the activity of double-stranded RNA-dependent protein kinase (PKR)-like ER kinase (PERK) and the activity of inositol-requiring enzyme 1 (IRE1) of ER stress biomarkers in quadriceps from the exhaustive swimming mice increased markedly compare to control mice. Furthermore, the gene expressions of GRP78, spliced X-box-binding protein 1 (XBP1s), C/EBP homologous protein (CHOP) and growth arrest and DNA damage-inducible protein (GADD34) of ER stress biomarkers in quadriceps from the exhaustive swimming mice enhanced significantly compare to control mice. The levels of blood glucose and muscle glycogen from the exhaustive swimming mice were significantly lower than those of control mice. A significant negative correlation was found between the muscle glycogen and each ER stress biomarker (Table). These results indicate that elevated ER stress biomarkers and lowered muscle glycogen may have important metabolic consequences in skeletal muscle.

\begin{tabular}{|c|c|c|c|c|c|c|c|c|}
\hline \multirow[b]{3}{*}{ Quadriceps } & \multicolumn{8}{|c|}{ Protein } \\
\hline & \multicolumn{3}{|c|}{ GRP78 } & \multicolumn{3}{|c|}{ PERK } & \multicolumn{2}{|c|}{ IRE1 } \\
\hline & & & $P$ & $r$ & & & $r$ & $P$ \\
\hline \multirow[t]{3}{*}{ Muscle glycogen } & & & $<0.01$ & -0.96 & & & -0.89 & $<0.01$ \\
\hline & \multicolumn{8}{|c|}{ Gene expression } \\
\hline & \multicolumn{2}{|c|}{ GRP78 } & \multicolumn{2}{|c|}{$\mathrm{XBP} 1 \mathrm{~s}$} & \multicolumn{2}{|c|}{ CHOP } & \multicolumn{2}{|c|}{ GADD34 } \\
\hline Quadriceps & $r$ & $P$ & $r$ & $P$ & $r$ & $P$ & $r$ & $P$ \\
\hline Muscle glycogen & -0.95 & $<0.01$ & -0.94 & $<0.01$ & -0.90 & $<0.01$ & -0.93 & $<0.01$ \\
\hline
\end{tabular}

1. Kitamura M (2008) Am J Physiol Renal Physiol 295, F323-F334.

2. Walter P \& Ron D (2011) Science 334, 1081-1086.

3. Deldicque L, Hespel P \& Francaux M (2012) Exerc Sport Sci Rev 40, 43-49. 